\title{
Conciliation and Consensus in Iterated Belief Merging
}

\author{
Olivier Gauwin, Sébastien Konieczny, and Pierre Marquis \\ CRIL - CNRS, Université d'Artois, 62300 Lens, France \\ \{gauwin, konieczny, marquis\}@eril.univ-artois.fr
}

\begin{abstract}
Two conciliation processes for intelligent agents based on an iterated merge-then-revise change function for belief profiles are introduced and studied. The first approach is skeptical in the sense that at any revision step, each agent considers that her current beliefs are more important than the current beliefs of the group, while the other case is considered in the second, credulous approach. Some key features of such conciliation processes are pointed out for several merging operators; especially, the convergence issue, the existence of consensus and the properties of the induced iterated merging operators are investigated.
\end{abstract}

\section{Introduction}

Belief merging is about the following question: given a set of agents whose belief bases are (typically) mutually inconsistent, how to define a belief base reflecting the beliefs of the group of agents? There are many different ways to address the belief merging issue in a propositional setting (see e.g.[11, 18, 16, 15, 2, 3, 13, 14]). The variety of approaches just reflects the various ways to deal with inconsistent beliefs.

The belief merging issue is not concerned with the way the result is exploited by the group. One possibility is to suppose that all the belief bases are replaced by the (agreed) merged base. This scenario is sensible with low-level agents that are used for distributed computation, or for applications with distributed information sources (like distributed databases). Once the merged base has been computed, all the agents participating to the merging process are equivalent in the sense that they share the same belief base. Such a drastic approach clearly leads to impoverish the beliefs of the system. Contrastingly, when high-level intelligent agents are considered, the previous scenario looks rather unlikely: it is not reasonable to assume that the agents are ready to completely discard their current beliefs and inconditionnally accept the merged base as a new belief base. It seems more adequate for them to incorporate the result of the merging process into their current belief base. Such an incorporation of new beliefs calls for belief revision $[1,7,8]$. In this perspective, two revision strategies can be considered. The first one consists in giving more priority to the previous beliefs; this is the strategy at work for skeptical agents. The second one, used by credulous agents, views the current beliefs of the group as more important than their own, current beliefs. Thus, given a revision strategy, every merging operator $\triangle$ induces what we call a conciliation operator which maps every belief profile $E$ (i.e., the beliefs associated to each agent at start) to a new belief profile where the new beliefs of an agent are obtained by confronting her previous beliefs with the merged base given by $E$ and $\triangle$.

Obviously enough, it makes sense to iterate such a merge-then-revise process when the aim of agents is to reach an agreement (if possible): after a first merge-then-revise

L. Godo (Ed.): ECSQARU 2005, LNAI 3571, pp. 514-526, 2005.

(C) Springer-Verlag Berlin Heidelberg 2005 
round, each agent has possibly new beliefs, defined from her previous ones and the beliefs of the group; this may easily give rise to new beliefs for the group, which must be incorporated into the previous beliefs of agents, and so on. The purpose of this paper is to study the two conciliation processes induced by the two revision strategies for various merging operators under two simplifying assumptions: homogeneity (the same revision operator is used by all the agents) and compatibility (the revision operator used is the one induced by the merging operator under consideration). Some key issues are considered, including the convergence of the processes (i.e., the existence of a round from which no further evolution is possible), the existence of consensus (i.e., the joint consistency of all belief bases at some stage), and the logical properties of the iterated merging operator defined by the last merged base once a fixed point has been reached.

The rest of the paper is organized as follows. In the next section, some formal preliminaries are provided. Section 3 presents the main results of the paper: in Section 3.1 the conciliation processes are defined, in Section 3.2 the focus is laid on the skeptical ones and in Section 3.3 on the credulous ones. In Section 4 we investigate the connections between the conciliation processes and the merging operators they induce. Especially, we give some properties of the corresponding iterated merging operators. Section 5 is devoted to related work. Finally, Section 6 gives some perspectives.

\section{Preliminaries}

We consider a propositional language $\mathcal{L}$ over a finite alphabet $\mathcal{P}$ of propositional symbols. An interpretation is a function from $\mathcal{P}$ to $\{0,1\}$. The set of all the interpretations is denoted $\mathcal{W}$. An interpretation $\omega$ is a model of a formula $K$, noted $\omega \models K$, if it makes it true in the usual classical truth functional way. Let $K$ be a formula, $[K]$ denotes the set of models of $K$, i.e., $[K]=\{\omega \in \mathcal{W} \mid \omega \models K\}$.

A belief base $K$ is a consistent propositional formula (or, equivalently, a finite consistent set of propositional formulas considered conjunctively), viewed up to logical equivalence.

Let $K_{1}, \ldots, K_{n}$ be $n$ belief bases (not necessarily pairwise different). We call belief profile the vector $E$ consisting of those $n$ belief bases in a specific order, $E=$ $\left(K_{1}, \ldots, K_{n}\right)$, so that the $n^{\text {th }}$ base gathers the beliefs of agent $n$. We note $\bigwedge E$ the conjunction of the belief bases of $E$, i.e., $\wedge E=K_{1} \wedge \cdots \wedge K_{n}$. We say that a belief profile $E$ is consistent if $\bigwedge E$ is consistent. The union operator for belief profiles (actually, of the associated multi-sets) will be noted $\sqcup$.

Let $\mathcal{E}$ be the set of all finite non-empty belief profiles. Two belief profiles $E_{1}$ and $E_{2}$ from $\mathcal{E}$ are said to be equivalent (noted $E_{1} \equiv E_{2}$ ) if and only if there is a bijection between the profile $E_{1}$ and the profile $E_{2}$ s.t. each belief base of $E_{1}$ is logically equivalent to its image in $E_{2}$. Note that the order given by the profile is not relevant for equivalence.

For every belief revision operator $*$, every profile $E=\left(K_{1}, \ldots, K_{n}\right)$ and every belief base $K$, we define the revision of $E$ by $K$ (resp. the revision of $K$ by $E$ ) as the belief profile given by $\left(K_{1}, \ldots, K_{n}\right) * K=\left(K_{1} * K, \ldots, K_{n} * K\right)$ (resp. $K *$ $\left.\left(K_{1}, \ldots, K_{n}\right)=\left(K * K_{1}, \ldots, K * K_{n}\right)\right)$. Since sequences of belief profiles will be considered, we use superscripts to denote belief profiles obtained at some stage, while subscripts are used (as before) to denote belief bases within a profile. For instance, $E^{i}$ 
denotes the belief profile obtained after $i$ elementary evolution steps (in our framework, $i$ merge-then-revise steps), and $K_{j}^{i}$ the belief base associated the the $j^{t h}$ coordinate of vector $E^{i}$ (i.e. the beliefs of agent $j$ at step $i$ ).

\subsection{IC Merging Operators}

Some basic work in belief merging aims at determining sets of axiomatic properties valuable operators should exhibit $[17,18,15,12,13,14]$. We focus here on the characterization of Integrity Constraints (IC) merging operators [13, 14].

The aim is to characterize the belief base $\triangle_{\mu}(E)$, that represents the merging of the profile $E$ under the integrity constraints $\mu$. $\mu$ is a formula that encodes some constraints on the result (such as physical constraints, laws, norms, etc...).

Definition 1. $\triangle$ is an IC merging operator if and only if it satisfies the following properties:

(IC0) $\triangle_{\mu}(E) \models \mu$

(IC1) If $\mu$ is consistent, then $\triangle_{\mu}(E)$ is consistent

(IC2) If $\wedge E$ is consistent with $\mu$, then $\triangle_{\mu}(E) \equiv \wedge E \wedge \mu$

(IC3) If $E_{1} \equiv E_{2}$ and $\mu_{1} \equiv \mu_{2}$, then $\triangle_{\mu_{1}}\left(E_{1}\right) \equiv \triangle_{\mu_{2}}\left(E_{2}\right)$

(IC4) If $K_{1} \models \mu$ and $K_{2} \models \mu$, then $\triangle_{\mu}\left(\left\{K_{1}, K_{2}\right\}\right) \wedge K_{1}$ is consistent if and only if

$\triangle_{\mu}\left(\left\{K_{1}, K_{2}\right\}\right) \wedge K_{2}$ is consistent

(IC5) $\triangle_{\mu}\left(E_{1}\right) \wedge \triangle_{\mu}\left(E_{2}\right) \models \triangle_{\mu}\left(E_{1} \sqcup E_{2}\right)$

(IC6) If $\triangle_{\mu}\left(E_{1}\right) \wedge \triangle_{\mu}\left(E_{2}\right)$ is consistent, then $\triangle_{\mu}\left(E_{1} \sqcup E_{2}\right) \models \triangle_{\mu}\left(E_{1}\right) \wedge \triangle_{\mu}\left(E_{2}\right)$

(IC7) $\triangle_{\mu_{1}}(E) \wedge \mu_{2} \models \triangle_{\mu_{1} \wedge \mu_{2}}(E)$

(IC8) If $\triangle_{\mu_{1}}(E) \wedge \mu_{2}$ is consistent, then $\triangle_{\mu_{1} \wedge \mu_{2}}(E) \models \triangle_{\mu_{1}}(E)$

For explanations on those properties see [14]. Two subclasses of IC merging operators have been defined. IC majority operators aim at resolving conflicts by adhering to the majority wishes, while IC arbitration operators exhibit a more consensual behaviour:

Definition 2. An IC majority operator is an IC merging operator which satisfies the following majority postulate:

(Maj) $\exists n \triangle_{\mu}(E_{1} \sqcup \underbrace{E_{2} \sqcup \ldots \sqcup E_{2}}_{n}) \models \triangle_{\mu}\left(E_{2}\right)$.

An IC arbitration operator is an IC merging operator which satisfies the following arbitration postulate:

$$
\left.\begin{array}{l}
\triangle_{\mu_{1}}\left(K_{1}\right) \equiv \triangle_{\mu_{2}}\left(K_{2}\right) \\
\triangle_{\mu_{1}} \Leftrightarrow \mu_{2}\left(\left\{K_{1}, K_{2}\right\}\right) \equiv\left(\mu_{1} \Leftrightarrow \neg \mu_{2}\right) \\
\mu_{1} \not \models \mu_{2} \\
\mu_{2} \not \forall \mu_{1}
\end{array}\right\} \Rightarrow \triangle_{\mu_{1} \vee \mu_{2}}\left(\left\{K_{1}, K_{2}\right\}\right) \equiv \triangle_{\mu_{1}}\left(K_{1}\right) .
$$

See $[13,14]$ for explanations about those two postulates and the behaviour of the two corresponding classes of merging operators. Let us now give some examples of IC merging operators.

Definition 3. A pseudo-distance $d$ between interpretations is a total function $d: \mathcal{W} \times \mathcal{W} \mapsto \mathbb{R}^{+}$such that for any $\omega, \omega^{\prime}, \omega^{\prime \prime} \in \mathcal{W}, d\left(\omega, \omega^{\prime}\right)=d\left(\omega^{\prime}, \omega\right)$, and $d\left(\omega, \omega^{\prime}\right)=0$ if and only if $\omega=\omega^{\prime}$. 
Two widely used pseudo-distances between interpretations are Dalal distance [6], denoted $d_{H}$, which is the Hamming distance between interpretations (i.e., the number of propositional variables on which the two interpretations differ); and the drastic distance, denoted $d_{D}$, which is the simplest pseudo-distance one can define: it gives 0 if the two interpretations are the same one, and 1 otherwise.

Definition 4. An aggregation function $f$ is a total function associating a nonnegative real number to every finite tuple of nonnegative real numbers and s.t. for any $x_{1}, \ldots, x_{n}, x, y \in \mathbb{R}^{+}$:

- if $x \leq y$, then $f\left(x_{1}, \ldots, x, \ldots, x_{n}\right) \leq f\left(x_{1}, \ldots, y, \ldots, x_{n}\right)$. (non-decreasingness)

$-f\left(x_{1}, \ldots, x_{n}\right)=0$ if and only if $x_{1}=\ldots=x_{n}=0 . \quad$ (minimality)

- $f(x)=x$.

(identity)

Widely used functions are the $\max [18,14]$, the sum $\Sigma[18,16,13]$, or the leximax GMax $[13,14]$. Then, given a distance $d$ and an aggregation function $f$, one can define a merging operator $\triangle^{d, f}$ :

Definition 5. Let $d$ be a pseudo-distance between interpretations and $f$ be an aggregation function. The result $\triangle_{\mu}^{d, f}(E)$ of the merging of $E$ given the integrity constraints $\mu$ is defined by:

- $d(\omega, K)=\min _{\omega^{\prime}=K} d\left(\omega, \omega^{\prime}\right)$.

- $d(\omega, E)=f_{\left\{K_{i} \in E\right\}}\left(d\left(\omega, K_{i}\right)\right)$.

- $\omega \leq_{E} \omega^{\prime}$ if and only if $d(\omega, E) \leq d\left(\omega^{\prime}, E\right)$.

- $\left[\triangle_{\mu}^{\bar{d}_{\mu}, f}(E)\right]=\min \left([\mu], \leq_{E}\right)$.

\subsection{Merging vs. Revision}

Belief revision operators can be viewed as special cases of belief merging operators when applied to singleton profiles, as stated below.

Theorem 1 ([14]). If $\triangle$ is an IC merging operator (it satisfies (ICO-IC8)), then the operator $* \triangle$, defined as $K * \triangle \mu=\triangle_{\mu}(K)$, is an AGM revision operator (it satisfies (R1-R6)) [8]. This operator is called the revision operator associated to the merging operator $\triangle$.

\section{Conciliation Operators}

Conciliation operators aim at reflecting the evolution of belief profiles, typically towards the achievement of some agreements between agents. It can be viewed as a simple form of negotiation, where the way beliefs may evolve is uniform.

\subsection{Definitions}

Let us first give the following, very general, definition of conciliation operators:

Definition 6. A conciliation operator is a function from the set of belief profiles to the set of belief profiles. 
This definition does not impose any strong constraints on the result, except that each resulting belief profile is solely defined from the given one. This does not prevent conciliation operators from taking advantage of additional information as parameters. For instance, integrity constraints representing norms or laws of nature can be taken into account. There are several ways to do it; if one assumes that agents must obey such laws, one can discard from the profile any agent who does not satisfy this requirement; one can also ask each agent to revise her own beliefs by the integrity constraints as a preliminary step so as to ensure it. In the following we adhere to a more liberal attitude and require integrity constraints to be satisfied at the group level, i.e. we do not ask that each agent satisfies the constraints. This relaxation is all the more important when conciliation is about preferences (i.e., goals): each agent is about to change her preferences in the light of the preferences of other agents, in the objective of achieving some agreements; each agent is free to have her own preferences, even if they are unfeasible. Nevertheless, the most preferred alternatives at the group level have to be feasible.

Clearly, pointing out the desirable properties for such conciliation operators is an interesting issue. We let this for future work, but one can note that the social contraction functions introduced by Booth [5] are very close to this idea.

In this paper we focus on a particular family of conciliation operators: conciliation operators induced by an iterated merge-then-revise process. The idea is to compute the belief merging from the profile, to revise the beliefs of each source by the result of the merging, and to repeat this process until a fixed point is reached. When such a fixed point exists, the conciliation operator is defined and the resulting profile is the image of the original profile by this operator.

When a fixed point has been reached, incorporating the beliefs of the group has no further impact on the own beliefs of each agent; in some sense, each agent did its best w.r.t. the group, given its revision function. Then there are two possibilities: either a consensus has been obtained, or no consensus can be obtained that way:

Definition 7. There is a consensus for a belief profile $E$ if and only if $E$ is consistent (with the integrity constraints).

The existence of a consensus for a belief profile just means that the associated agents agree on at least one possible world. When this is the case, the models of the corresponding merged base w.r.t. any IC merging operator reduce to such possible worlds ((IC2) ensures it). Interestingly, it can be shown that the existence of a consensus at some stage of the merge-then-revise process is sufficient to ensure the existence of a fixed point, hence the termination of the process. Let us now consider two additional properties on conciliation operators in order to keep the framework simple enough: homogeneity and compatibility.

Definition 8. Let $\triangle$ be a revision operator, and let $*_{1}, \ldots, *_{n}$ be $n$ revision operators. An iterated merging conciliation operator is a function from the set of belief profiles to the set of belief profiles, where the evolution of a profile is characterized by a mergethen-revise approach. It is:

- homogeneous if all the agents use the same revision operator $*_{1}=\ldots=*_{n}=*$,

- compatible if the revision operator is associated to the merging operator $*=* \triangle$.

In this work, we focus on compatible homogeneous iterated merging conciliation operators (CHIMC in short). Under the compatibility and homogeneity assumptions, 
defining a CHIMC operator just requires to make precise the belief merging operator under use and the revision strategy (skeptical or credulous):

Definition 9. Let $\triangle$ be an IC merging operator, and $*$ its associated revision operator (i.e., $\left.\varphi * \mu=\triangle_{\mu}(\{\varphi\})\right)$. Let $E$ be any belief profile. We define the sequence $\left(E_{s}^{i}\right)_{i}$ (depending on both $\triangle$ and $E$ ) by:

$-E_{s}^{0}=E$,

$$
\text { - } E_{s}^{i+1}=\triangle_{\mu}\left(E_{s}^{i}\right) * E_{s}^{i}
$$

The skeptical CHIMC operator induced by $\triangle$ is defined by $\triangle_{\mu}^{*}(E)=E_{s}^{k}$, where $k$ is the lowest rank $i$ such that $E_{s}^{i}=E_{s}^{i+1}$, and $\triangle_{\mu}^{*}(E)$ is undefined otherwise. We note $E_{s}^{*}=E_{s}^{k}$ the resulting profile.

Definition 10. Let $\triangle$ be an IC merging operator, and $*$ its associated revision operator. Let $E$ be any belief profile. We define the sequence $\left(E_{c}^{i}\right)_{i}$ by:

$-E_{c}^{0}=E$,

$$
\text { - } E_{c}^{i+1}=E_{c}^{i} * \triangle_{\mu}\left(E_{c}^{i}\right)
$$

The credulous CHIMC operator induced by $\triangle$ is defined by ${ }^{*} \triangle_{\mu}(E)=E_{c}^{k}$, where $k$ is the lowest rank $i$ such that $E_{c}^{i}=E_{c}^{i+1}$, and ${ }^{*} \triangle_{\mu}(E)$ is undefined otherwise. We note $E_{c}^{*}=E_{c}^{k}$ the resulting profile.

Every CHIMC operator induces a merging operator: the operator that associates to each profile the merged base of the resulting profile. Formally:

Definition 11. Let $\triangle$ be an IC merging operator, and $*$ its associated revision operator.

- The skeptical CHIM operator induced by $\triangle$ is the function that maps every profile $E$ to $\triangle_{\mu}\left(\triangle_{\mu}^{*}(E)\right)$.

- The credulous CHIM operator induced by $\triangle$ is the function that maps every profile E to $\triangle_{\mu}\left({ }^{*} \triangle_{\mu}(E)\right)$.

Let us now study the key features of the two sequences $\left(E_{s}^{i}\right)_{i}$ and $\left(E_{c}^{i}\right)_{i}$ and the properties of the corresponding iterated merging operators, based on various IC merging operators.

\subsection{Skeptical Operators}

We start with skeptical CHIMC operators. Let us first give an important monotony property, which states that the conciliation process given by any IC merging operator $\triangle$ may only lead to strengthen the beliefs of each agent:

Theorem 2. Let $K_{j}^{i}$ denote the belief base corresponding to agent $j$ in the belief profile $E_{s}^{i}$ characterized by the initial belief profile $E$ and the IC merging operator $\triangle$. For every $i, j$, we have $K_{j}^{i+1} \models K_{j}^{i}$.

On this ground, it is easy to prove that the sequence $\left(E_{s}^{i}\right)_{i}$ is stationary at some stage, for every profile $E$ and every IC merging operator $\triangle$. Accordingly, the induced skeptical conciliation operator and the induced skeptical iterated merging operator are defined for every $E$ : 
Theorem 3. For every belief profile $E$ and every IC merging operator $\triangle$, the stationarity of $\left(E_{s}^{i}\right)_{i}$ is reached at a rank bounded by $\left(\sum_{K \in E} \#([K))-\#(E)\right.$. Therefore, the CHIMC operator $\triangle^{*}$ and the CHIM operator $\triangle\left(\triangle^{*}\right)$ are total functions.

The bound on the number of iterations is easily obtained from the monotony property. Another interesting property is that the sequence of profiles and the corresponding sequence of merged bases are equivalent with respect to stationarity:

Theorem 4. Let $E$ be a belief profile and $\triangle$ be an IC merging operator. Let $\mu$ be any integrity constraint. The sequence $\left(E_{s}^{i}\right)_{i}$ is stationary from some stage if and only if the sequence $\left(\triangle_{\mu}\left(E_{s}^{i}\right)\right)_{i}$ is stationary from some stage.

The number of iterations needed to reach the fixed point of $\left(E_{s}^{i}\right)_{i}$ is one for the IC merging operators defined from the drastic distance. More precisely, the skeptical CHIM operator induced by any IC merging operator $\triangle$ defined from the drastic distance coincides with $\triangle$.

Theorem 5. Let $E=\left(K_{1}, \ldots, K_{n}\right)$ be a profile. If the IC merging operator $\triangle$ is among $\triangle^{d_{D}, \text { Max }}, \triangle^{d_{D}, \Sigma}, \triangle^{d_{D}, G \text { Max }}$, then for every $j$, the base $K_{j}^{*}$ from the resulting profile $E^{*}=\triangle_{\mu}^{*}(E)$ can be characterized by:

$$
K_{j}^{*}= \begin{cases}\mu \wedge \triangle_{\mu}(E) & \text { if consistent, else } \\ \triangle_{\mu}(E) & \text { otherwise. }\end{cases}
$$

Furthermore, the resulting profile is obtained after at most one iteration (i.e., for every $\left.i>0, E^{i}=E^{i+1}\right)$.

We have no direct (i.e., non-iterative) definition for any skeptical CHIM operator based on an IC merging operator defined from Dalal distance. Let us give an example of such an operator.

Example 1. Let us consider the profile $E=\left(K_{1}, K_{2}, K_{3}\right)$ with $\left[K_{1}\right]=\{(0,0,0)$, $(0,0,1),(0,1,0)\},\left[K_{2}\right]=\{(0,1,1),(1,1,0),(1,1,1)\},\left[K_{3}\right]=\{(0,0,0),(1,0,0)$, $(1,0,1),(1,1,1)\}$, no integrity constraints $(\mu \equiv \mathrm{T})$, and the skeptical CHIMC operator defined from the $\triangle^{d_{H}, G M a x}$ operator. The complete process is represented in Table 1 . The first three columns show the Dalal distance between each interpretation and the corresponding source. The last column shows the distance between each interpretation and the profile according to the aggregation function. So the selected interpretations for the corresponding operators are the ones with minimal aggregated distance. As there are several (three in that case) iterations, we sum up the three tables (corresponding to the three merging steps) in the same one. So, for example in column $d\left(\omega, K_{1}^{i}\right)$, the first number denotes the distance between the interpretation $\omega$ and $K_{1}^{1}$, the second one the distance between $\omega$ and $K_{1}^{2}$, etc.

Let us explain the full process in details. The first profile is $E^{0}=E$. The first merging iteration gives as result $\left[\triangle^{d_{H}, G M a x}\left(E^{0}\right)\right]=\{(0,0,1),(0,1,0),(0,1,1),(1,0,0)$, $(1,0,1),(1,1,0)\}$. Then, every source revises the result of the merging with its old beliefs, i.e., $K_{i}^{1}=\triangle^{d_{H}, \operatorname{GMax}}\left(E^{0}\right) * K_{i}^{0}$, so $\left[K_{1}^{1}\right]=\{(0,0,1),(0,1,0)\},\left[K_{2}^{1}\right]=\{(0,1,1)$, $(1,1,0)\}$ and $\left[K_{3}^{1}\right]=\{(1,0,0),(1,0,1)\}$. Since each of the three bases is consistent with the merged base, the new base of each agent is just the conjunction of her previous base with the merged base (in accordance to revision postulates). Then, the second 
Table 1. $\triangle_{\mu}^{*} d_{H}, G M a x$

\begin{tabular}{c|ccc|c}
$\omega$ & $d\left(\omega, K_{1}^{i}\right) d\left(\omega, K_{2}^{i}\right) d\left(\omega, K_{3}^{i}\right)$ & $d_{\text {GMax }}\left(\omega, E^{i}\right)_{d\left(\omega, \triangle_{\mu}\left(E^{i}\right)\right)}$ \\
\hline$(0,0,0)$ & $0,1,1$ & $2,2,2$ & $0,1,1$ & $(2,0,0)_{1},(2,1,1)_{1},(2,1,1)_{1}$ \\
$(0,0,1)$ & $0,0,0$ & $1,1,3$ & $1,1,1$ & $(1,1,0)_{0},(1,1,0)_{0},(3,1,0)_{1}$ \\
$(0,1,0)$ & $0,0,2$ & $1,1,1$ & $1,2,2$ & $(1,1,0)_{0},(2,1,0)_{1},(2,2,1)_{2}$ \\
$(0,1,1)$ & $1,1,1$ & $0,0,2$ & $1,2,2$ & $(1,1,0)_{0},(2,1,0)_{1},(2,2,1)_{2}$ \\
$(1,0,0)$ & $1,2,2$ & $1,1,1$ & $0,0,0$ & $(1,1,0)_{0},(2,1,0)_{1},(2,1,0)_{0}$ \\
$(1,0,1)$ & $1,1,1$ & $1,2,2$ & $0,0,0$ & $(1,1,0)_{0},(2,1,0)_{1},(2,1,0)_{0}$ \\
$(1,1,0)$ & $1,1,3$ & $0,0,0$ & $1,1,1$ & $(1,1,0)_{0},(1,1,0)_{0},(3,1,0)_{1}$ \\
$(1,1,1)$ & $2,2,2$ & $0,1,1$ & $0,1,1$ & $(2,0,0)_{1},(2,1,1)_{1},(2,1,1)_{1}$
\end{tabular}

merging iteration gives $\left[\triangle^{d_{H}, G \operatorname{Max}}\left(E^{1}\right)\right]=\{(0,0,1),(1,1,0)\}$, and the revision of each base gives $\left[K_{1}^{2}\right]=\{(0,0,1)\},\left[K_{2}^{2}\right]=\{(1,1,0)\}$, and $\left[K_{3}^{2}\right]=\{(1,0,0),(1,0,1)\}$. The third iteration step gives $\left[\triangle^{d_{H}, G \operatorname{Max}}\left(E^{2}\right)\right]=\{(1,0,0),(1,0,1)\}$, and the revision step does not change any belief base, i.e., $E^{2} \equiv E^{3}$, so a stationary point is reached and the process stops on this profile.

As to skeptical operators, the conciliation process cannot lead to a consensus, unless a consensus already exists at start:

Theorem 6. Let $E$ be a belief profile and $\triangle$ be an IC merging operator. There exists a rank $i$ s.t. a consensus exists for $E_{s}^{i}$ if and only if $i=0$ and there is a consensus for $E$.

\subsection{Credulous Operators}

Let us now turn to credulous CHIMC operators. Let us first give some general properties about credulous operators.

Theorem 7. Let $K_{j}^{i}$ now denote the belief base corresponding to agent $j$ in the belief profile $E_{c}^{i}$ characterized by the initial belief profile $E$ and the IC merging operator $\triangle$.

- $\forall i, j K_{j}^{i+1} \models \triangle_{\mu}\left(E_{c}^{i}\right)$,

- $\forall i>0 \forall j K_{j}^{i} \models \mu$,

- $\forall i, j$, if $K_{j}^{i} \wedge \triangle_{\mu}\left(E_{c}^{i}\right)$ is consistent, then $K_{j}^{i+1} \equiv K_{j}^{i} \wedge \triangle_{\mu}\left(E_{c}^{i}\right)$.

The first item states that, during the evolution process, each base implies the previous merged base. The second item states that from the first iteration, all the bases implies the integrity constraints. The last one is simply a consequence of a revision property: if, at a given step, a base is consistent with the result of the merging, then the base at the next step will be that conjunction.

Unfortunately, the monotony property as reported in Theorem 2 does not hold in the credulous case. At that point, we can just conjecture that our credulous CHIMC operators (and the corresponding iterated merging operators) are defined for every profile:

Conjecture 1. For every belief profile $E$ and every merging operator $\triangle$ using the aggregation function Max, GMax or $\Sigma$, the sequence $\left(E_{c}^{i}\right)_{i}$ is stationary from some rank. 
This claim is supported by some empirical evidence. We have conducted exhaustive tests for profiles containing up to three bases, when the set of propositional symbols contains up to three variables. The following IC merging operators have been considered: $\triangle^{d_{H}, M a x}, \triangle^{d_{H}, G M a x}$ and $\triangle^{d_{H}, \Sigma}$. We have also conducted non-exhaustive tests when four propositional symbols are considered in the language (this leads to billions of tests). All the tested instances support the claim (stationarity is reached in less than five iterations when up to three symbols are considered, and less than ten iterations when four symbols are used).

We can nevertheless prove the stationarity of $\left(E_{c}^{i}\right)_{i}$ for every belief profile $E$ when some specific IC merging operators $\triangle$ are considered. In particular, for IC merging operators defined from the drastic distance, it is possible to find out a non-iterative definition of the corresponding CHIMC operator, and to prove that it is defined for every profile.

Theorem 8. Let $E=\left(K_{1}, \ldots, K_{n}\right)$ be a profile. If the IC merging operator is $\triangle^{d_{D}, \text { Max }}$, then for every $j$, the base $K_{j}^{*}$ from the resulting profile $E^{*}={ }^{*} \triangle_{\mu}^{d_{D}, \text { Max }}(E)$ can be characterized by:

$$
K_{j}^{*}= \begin{cases}\mu \wedge \bigwedge_{K_{i}: K_{i} \wedge \mu \nvdash \perp} K_{i} \text { if consistent, else } \\
\mu \wedge K_{j} & \begin{array}{l}
\text { if consistent, else } \\
\mu
\end{array} \\
\text { otherwise. }\end{cases}
$$

Furthermore, the resulting profile is obtained after at most two iterations (i.e., for every $i>1, E^{i}=E^{i+1}$ ).

Theorem 9. Let $E=\left(K_{1}, \ldots, K_{n}\right)$ be a profile. If the IC merging operator is $\triangle^{d_{D}, G M a x}$ or $\triangle^{d_{D}, \Sigma}$, then for every $j$, the base $K_{j}^{*}$ from the resulting profile $E^{*}={ }^{*} \triangle_{\mu}^{d_{D}, G M a x}(E)=$ $* \triangle_{\mu}^{d_{D}, \Sigma}(E)$ can be characterized by:

$$
K_{j}^{*}= \begin{cases}K_{j} \wedge \triangle_{\mu}^{d_{D}, G M a x}(E) & \text { if consistent, else } \\ \triangle_{\mu}^{d_{D}, G M a x}(E) & \text { otherwise. }\end{cases}
$$

Furthermore, the resulting profile is obtained after at most one iteration (i.e., for every $\left.i>0, E^{i}=E^{i+1}\right)$.

Finally, like for the skeptical case, the sequence of profiles and the corresponding sequence of merged bases are equivalent w.r.t. stationarity in the credulous case:

Definition 12. Let $E$ be a belief profile and $\triangle$ be an IC merging operator. Let $\mu$ be any integrity constraint. The sequence $\left(E_{s}^{i}\right)_{i}$ is stationary from some stage if and only if the sequence $\left(\triangle_{\mu}\left(E_{s}^{i}\right)\right)_{i}$ is stationary from some stage.

Let us consider an example of credulous operator at work.

Example 2. Consider the profile $E=\left(K_{1}, K_{2}, K_{3}, K_{4}\right)$, with $\left[K_{1}\right]=\{(0,0,0),(0,0$, $1),(0,1,0)\},\left[K_{2}\right]=\{(1,0,0),(1,0,1),(1,1,1)\},\left[K_{3}\right]=\{(0,0,1),(0,1,0),(0,1,1)$, $(1,1,0)\},\left[K_{4}\right]=\{(0,1,1),(1,0,0),(1,1,0),(1,1,1)\}$. There is no integrity constraint $\mu \equiv \top$. Let us consider the credulous CHIMC operator defined from the merging 
Table 2. ${ }^{*} \triangle_{\mu}^{d_{H}, \Sigma}$

\begin{tabular}{c|cccc|c}
$\omega$ & $d\left(\omega, K_{1}^{i}\right)$ & $d\left(\omega, K_{2}^{i}\right)$ & $d\left(\omega, K_{3}^{i}\right)$ & $d\left(\omega, K_{4}^{i}\right)$ & $d_{\Sigma}\left(\omega, E^{i}\right)$ \\
\hline$(0,0,0)$ & $0,1,1$ & $1,1,1$ & $1,1,1$ & $1,1,1$ & $3,4,4$ \\
$(0,0,1)$ & $0,0,0$ & $1,2,2$ & $0,0,0$ & $1,1,2$ & $2,3,4$ \\
$(0,1,0)$ & $0,2,2$ & $2,2,2$ & $0,1,2$ & $1,1,2$ & $3,6,8$ \\
$(0,1,1)$ & $1,1,1$ & $1,3,3$ & $0,0,1$ & $0,0,3$ & $2,4,8$ \\
$(1,0,0)$ & $1,2,2$ & $0,0,0$ & $1,1,2$ & $0,0,0$ & $2,3,4$ \\
$(1,0,1)$ & $1,1,1$ & $0,1,1$ & $1,1,1$ & $1,1,1$ & $3,4,4$ \\
$(1,1,0)$ & $1,3,3$ & $1,1,1$ & $0,0,3$ & $0,0,1$ & $2,4,8$ \\
$(1,1,1)$ & $2,2,2$ & $0,2,2$ & $1,1,2$ & $0,1,2$ & $3,6,8$
\end{tabular}

operator $\triangle^{d_{H}, \Sigma}$. The computations are summarized in Table 2. The resulting profile is $\left[K_{1}^{2}\right]=\{(0,0,1)\},\left[K_{2}^{2}\right]=\{(1,0,0)\},\left[K_{3}^{2}\right]=\{(0,0,1)\}$ and $\left[K_{4}^{2}\right]=\{(1,0,0)\}$. And the corresponding CHIM operator gives as result a base whose models are $\{(0,0,0)$, $(0,0,1),(1,0,0),(1,0,1)\}$, that is different from the result of the merging of $E$ by the IC merging operator $\left[\triangle^{d_{H}, \Sigma}(E)\right]=\{(0,0,1),(0,1,1),(1,0,0),(1,1,0)\}$.

\section{Iterated Merging Operators}

An interesting question is to investigate the properties of the CHIM operators. A first important question is whether such operators are IC merging operators. The answer is negative in general, only some basic postulates are guaranteed to hold:

Theorem 10. Credulous and skeptical CHIM operators satisfy (ICO)-(IC3), (IC7) and (IC8).

Thus, some important properties of IC merging operators are usually lost through the merge-then-revise process. We claim that this is not so dramatic since the main purpose of conciliation processes is not exactly the one of belief merging. Furthermore, specific iterated merging operators (i.e., those induced by some specific merging operators $\triangle$ ) may easily satisfy additional postulates:

Theorem 11. The credulous iterated merging operator associated to ${ }^{*}{ }_{\mu}^{d_{D}, M a x}$ satisfies (ICO)-(IC5), (IC7)-(IC8) and (Arb). It satisfies neither (IC6) nor (Maj).

In fact, the CHIM operator defined from ${ }^{*} \triangle_{\mu}^{d_{D}, \text { Max }}$ can be defined as follows (this is a straightforward consequence of Theorem 8 ):

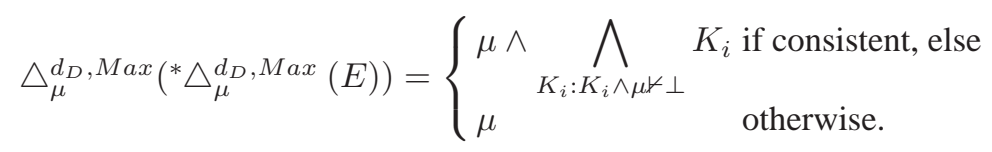

Theorem 12. The credulous iterated operator associated to ${ }^{*} \triangle_{\mu}^{d_{D}, G M a x}={ }^{*} \triangle_{\mu}^{d_{D}, \Sigma}$ satisfies (ICO)-(IC8), (Arb) and (Maj).

This result easily comes from the fact that this credulous CHIM operator actually coincides with the IC merging operator $\triangle_{\mu}^{d_{D}, G M a x}=\triangle_{\mu}^{d_{D}, \Sigma}$ it is based on. 
Thus, as for skeptical operators (see Theorem 5), each CHIM operator based on the drastic distance coincides with the underlying IC merging operator, so it satisfies exactly the same properties (see [14]).

As to the operators based on Dalal distance, things are less easy. Up to now, we did not find an equivalent, non-iterative, definition for any of them. We group the following results on credulous/skeptical operators since they satisfy the same properties, but the proofs of the results are different for the two kinds of operators. Furthermore, since stationarity is only conjectured for credulous operators (cf. Conjecture 1), we do not have a proof that the corresponding CHIM operators are total functions. So the two following results on credulous operators are guaranteed under the conjecture of stationarity, only.

Theorem 13. The credulous (resp. skeptical) CHIM operator associated to ${ }^{*} \triangle_{\mu}^{d_{H}, \Sigma}$ (resp. $\left.\triangle_{\mu}^{*} d_{H}, \Sigma\right)$ satisfies (IC0)-(IC3), (IC7)-(IC8) and (Maj), but does not satisfy (IC5)(IC6) and (Arb). The satisfaction of (IC4) is an open issue.

Theorem 14. The credulous (resp. skeptical) CHIM operators associated to ${ }^{*} \triangle_{\mu}^{d_{H}, \text { Max }}$

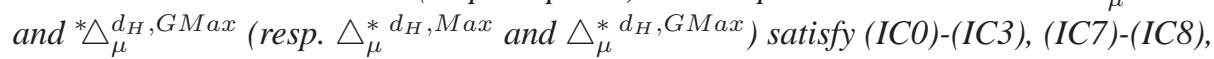
but satisfy none of (IC5)-(IC6), (Maj) and (Arb). The satisfaction of (IC4) is an open issue.

\section{Related Work}

In [5,4] Richard Booth presents what he calls Belief Negotiation Models. Such negotiation models can be formalized as games between sources: until a coherent set of sources is reached, at each round a contest is organized to find out the weakest sources, then those sources have to be logically weakened. This idea leads to numerous new interesting operators (depending of the exact meaning of "weakest" and "weaken", which correspond to the two parameters for this family). Booth is interested at the same time in the evolution of the profile (in connection to what he calls social contraction), and to the resulting merged base (the result of the Belief Negotiation Model).

In [10,9] a systematic study of a subclass of those operators, called Belief Game Models, is achieved. This subclass contains operators closer to merging ones than the general class which also allows negotiation-like operators.

All those operators are close in spirit to the CHIMC/CHIM operators defined in this work. A main difference is that in the work presented in this paper, the evolution of a profile does not always lead to a consensus. Scenarios where agents disagree at a final stage are allowed. Whereas in the former work, the evolution process leads to consensus (in fact consensus is the halting condition of the iterative definition). So CHIMC operators seem more adequate to formalize interaction between agents' beliefs. Thus, they are closer to negotiation processes, since the agents' beliefs change due to the interaction with other agents' beliefs, but this interaction can be stopped when the agents have achieved the best possible compromise. 


\section{Conclusion and Perspectives}

In this paper, we have introduced two conciliation processes based on an iterated mergethen-revise change function for the beliefs of agents. On this ground, a family of conciliation operators and an associated family of iterated merging operators have been defined and studied.

This work calls for several perspectives. One of them concerns the stationarity conjecture related to credulous CHIMC operators. A second perspective is about rationality postulates for conciliation operators; such postulates should reflect the fact that at the end of the conciliation process, the disagreement between the agents participating to the conciliation process is expected not to be more important than before; a difficulty is that it does not necessarily mean that this must be the case at each step of a conciliation process.

Furthermore, when a consensus is reached for those conciliation operators, one can use the number of steps needed to reach the consensus as a measure of conflict of the profiles. Such a measure could be used to compare several profiles and to determine what are the less conflictual ones.

Another perspective is to enrich our framework in several directions; one of them consists in relaxing the homogeneity assumption; in some situations, it can prove sensible to consider that an agent is free to reject a negotiation step, would it lead her to a belief state "too far" from its original one; another direction is to study less drastic revision behaviours, for example obtained through non-prioritized belief revision operators.

\section{Acknowledgements}

We would like to thank the anonymous reviewers for many helpful comments. The authors have been supported by the Université d'Artois, the Région Nord/Pas-de-Calais, the IRCICA consortium, and by the European Community FEDER Program.

\section{References}

1. C. E. Alchourrón, P. Gärdenfors, and D. Makinson. On the logic of theory change: Partial meet contraction and revision functions. Journal of Symbolic Logic, 50:510-530, 1985.

2. C. Baral, S. Kraus, and J. Minker. Combining multiple knowledge bases. IEEE Transactions on Knowledge and Data Engineering, 3(2):208-220, 1991.

3. C. Baral, S. Kraus, J. Minker, and V. S. Subrahmanian. Combining knowledge bases consisting of first-order theories. Computational Intelligence, 8(1):45-71, 1992.

4. R. Booth. A negociation-style framework for non-prioritised revision. In Proceedings of the Eighth Conference on Theoretical Aspects of Rationality and Knowledge (TARK'01), pages 137-150, 2001.

5. R. Booth. Social contraction and belief negociation. In Proceedings of the Eighth Conference on Principles of Knowledge Representation and Reasoning (KR'02), pages 374-384, 2002.

6. M. Dalal. Investigations into a theory of knowledge base revision: preliminary report. In Proceedings of the Seventh American National Conference on Artificial Intelligence (AAAI'88), pages $475-479,1988$. 
7. P. Gärdenfors. Knowledge in flux. MIT Press, 1988.

8. H. Katsuno and A. O. Mendelzon. Propositional knowledge base revision and minimal change. Artificial Intelligence, 52:263-294, 1991.

9. S. Konieczny. Belief base merging as a game. Journal of Applied Non-Classical Logics, 14(3):275-294, 2004.

10. S. Konieczny. Propositional belief merging and belief negotiation model. In Proceedings of the International Workshop on Non-Monotonic Reasoning (NMR'04), pages 249-257, 2004.

11. S. Konieczny, J. Lang, and P. Marquis. $\mathrm{DA}^{2}$ merging operators. Artificial Intelligence, 157(1-2):49-79, 2004.

12. S. Konieczny and R. Pino Pérez. On the logic of merging. In Proceedings of the Sixth International Conference on Principles of Knowledge Representation and Reasoning (KR'98), pages 488-498, 1998.

13. S. Konieczny and R. Pino Pérez. Merging with integrity constraints. In Proceedings of the Fifth European Conference on Symbolic and Quantitative Approaches to Reasoning with Uncertainty (ECSQARU'99), LNAI 1638, pages 233-244, 1999.

14. S. Konieczny and R. Pino Pérez. Merging information under constraints: a qualitative framework. Journal of Logic and Computation, 12(5):773-808, 2002.

15. P. Liberatore and M. Schaerf. Arbitration (or how to merge knowledge bases). IEEE Transactions on Knowledge and Data Engineering, 10(1):76-90, 1998.

16. J. Lin and A. O. Mendelzon. Knowledge base merging by majority. In Dynamic Worlds: From the Frame Problem to Knowledge Management. Kluwer, 1999.

17. P. Z. Revesz. On the semantics of theory change: arbitration between old and new information. In Proceedings of the Twelfth ACM SIGACT-SIGMOD-SIGART Symposium on Principles of Databases, pages 71-92, 1993.

18. P. Z. Revesz. On the semantics of arbitration. International Journal of Algebra and Computation, 7(2):133-160, 1997. 